Décadrages Décadrages

cınéma, à travers champs Cinéma, à travers champs

43 | 2020

Abdellatif Kechiche

\title{
La représentation de la masculinité dans les films américains ou comment dépasser le clivage entre les genres?
}

Compte rendu de lecture Des machines et des hommes : masculinité et technologie dans le cinéma américain contemporain de Charles-Antoine Courcoux, Genève, Georg éditeur, 2017

Jean-Michel Baconnier

\section{(2) OpenEdition}

Journals

Édition électronique

URL : https://journals.openedition.org/decadrages/1613

DOI : 10.4000/decadrages. 1613

ISSN : 2297-5977

Éditeur

Association Décadrages

Édition imprimée

Date de publication : 1 juillet 2020

Pagination : 192-197

ISSN : 2235-7823

\section{Référence électronique}

Jean-Michel Baconnier, «La représentation de la masculinité dans les films américains ou comment dépasser le clivage entre les genres ? », Décadrages [En ligne], 43 | 2020, mis en ligne le 15 octobre 2021, consulté le 06 mars 2022. URL : http://journals.openedition.org/decadrages/1613 ; DOI : https://doi.org/10.4000/decadrages.1613 
Des machines et des hommes

Charles-Antoine Courcoux

georg 


\section{LA REPRÉSENTATION DE LA MASCULINITÉ DANS LES FILMS AMÉRICAINS OU COMMENT DÉPASSER LE CLIVAGE ENTRE LES GENRES?}

\author{
Compte rendu de lecture \\ Des machines et des hommes: masculinité et technologie \\ dans le cinéma américain contemporain \\ de Charles-Antoine Courcoux, Genève, Georg éditeur, 2017
}

1 Voir Clevi Elena Rapkiewicz, «Femmes et métiers de l'informatique", Cahiers du GEDISST (Groupe d'étude sur la division sociale et sexuelle du travail), $n^{\circ} 19,1997$ («Travail, espaces et professions "), pp. 27-47.
Cet ouvrage de Charles-Antoine Courcoux est la version publiée, en 2017, de sa thèse de doctorat, chez Georg éditeur à Genève. Nous avons donc affaire à un épais volume de plus de cinq cents pages largement référencées et illustrées. L'ampleur de ce format donne à cette publication une dimension encyclopédique qui semble correspondre à un souci d'exhaustivité académique. Dans ce sens, l'ouvrage répond avec rigueur à l'idée d'un résultat détaillé d'une recherche de longue haleine aboutissant à une somme d'analyses argumentées, au service de l'histoire et de l'esthétique du cinéma. Cette approche disciplinaire se tisse à la croisée de l'étude des genres cinématographiques et des gender studies.

Courcoux se propose ainsi d'étudier les rapports ambigus entre l'identité masculine et la technologie dans le contexte de la production cinématographique hollywoodienne à l'ère postindustrielle. Par le biais de sa recherche, il tente d'expliquer l'inclination des scénarios d'un grand nombre de films populaires américains à envisager la technologie de manière avilissante alors qu'elle est elle-même au cœur de l'évolution de l'esthétique et de l'industrie du cinéma. Ainsi, il postule que le cinéma outre-Atlantique construit ses codes sociaux et ses canons esthétiques de la masculinité à contre-pied de l'évolution de la technologie et de la science. Cet antagonisme semble dès lors pointer un conflit de valeurs chez l'homme occidental qui va chercher à réaffirmer le pouvoir hégémonique de sa masculinité "déclinante». Le développement sans commune mesure de la technologie de la communication et de l'information, parallèlement à l'émergence de la visibilité des minorités sexuelles à partir des années 1960, sera perçu comme une menace de disqualification sociale de la puissance masculine.

Courcoux aurait d'ailleurs pu étendre son propos à ce sujet en revenant sur le rôle majeur qu'ont eu les femmes dans l'évolution des technologies de l'information, dès le $\mathrm{XIX}^{\mathrm{e}}$ siècle ${ }^{1}$. En effet, malgré leur part active dans le développement du domaine de l'informatique, cela n'a pas empêché leur éviction de I'histoire de l'innovation de cette technologique connue du grand public. Selon Isabelle 
2 Voir Isabelle Collet, Les Oubliées du numérique, Paris, Le Passeur Editeur, 2019. Idem, pp. 79-80.

Alan Kay cité par Patrick Flichy dans L'Imaginaire d'Internet, Paris, Editions La Découverte, 2001, p. 81.
Collet, cette disparition a eu lieu majoritairement après les années 1960, période charnière à laquelle le logiciel prend de la valeur au niveau économique et social en raison du développement exponentiel de l'informatique ${ }^{2}$. Par conséquent, si l'homme a pris l'évolution de la technologie numérique comme une menace envers l'image de sa masculinité, sa réponse fut de l'annexer à part entière pour l'assimiler à son genre. Cette idée de "remettre les choses en ordre", nous la trouvons au sein même de la définition de l'«ordinateur», terme proposé par le philologue Jacques Perret au responsable du service de la publicité chez IBM, en 1955, comme le rappelle Collet ${ }^{3}$. En effet, à I'origine, "ordinateur " est un adjectif désignant, selon le Littré, I'action de Dieu lorsqu'il met de l'ordre dans le monde. Nous retrouvons cette approche à travers la capacité qu'aurait I'homme à être le démiurge des mondes qu'il peut créer grâce à l'informatique au sein même des films et des jeux vidéo qu'il produit. Cette "méta" position du concepteur de mondes est justement possible avec l'arrivée de la numérisation des images à la fin des années 1970. Ainsi, I'homme devient le créateur de son propre environnement et peut le rendre accessible à toutes et tous par sa représentation et sa configuration informatisées. Durant cette même décennie, Alan Kay, chercheur à Xerox Parc, I'un des créateurs du micro-ordinateur, ira même jusqu'à avancer que "l'informatique met la science à l'envers. Dans la science normale, on vous donne un monde et votre travail est de trouver les règles qui s'appliquent. Dans l'informatique, vous donnez les règles à l'ordinateur et il crée le monde. " ${ }^{4}$ Au début des années 1980, Kay devient le responsable scientifique chez Atari qui avait pour slogan: "Atari, nous sommes tous des dieux". Nous retrouvons cette idée dans Tron (E.-U., 1982) de Steven Lisberger qui fait de cette posture théologique du concepteur de jeux vidéo un élément central dans le scénario du film.

C'est peut-être pour une part cette mise en abyme du créateur de mondes au sein même de l'environnement diégétique - comme lieu de la représentation de la dépréciation de l'hégémonie de la masculinité dans sa confrontation avec la technologie - qui permet paradoxalement que la technophobie des récits dans le cinéma hollywoodien puisse laisser une place à l'avènement de la technophilie des images cinématographiques et des effets spéciaux. Cette hypothèse aurait pu servir de piste, parmi d'autres, à l'auteur de Des machines et des hommes afin d'élargir son approche principalement dualiste entre la masculinité et la féminité, avant d'attendre la conclusion de son ouvrage pour l'envisager.

Le spectre historique retenu par Courcoux balaye justement un corpus de films emblématiques allant de la fin des années 1960 jusqu'à aujourd'hui. Les films traités proviennent principalement de deux genres cinématographiques: la science-fiction et le film catastrophe. Toutefois, I'historien en cinéma ne se borne pas à ces deux genres et en intègre d'autres à son étude comme le péplum, le film d'espionnage et le biopic. Parmi les nombreuses productions analysées, qui sont indexées à la fin de l'ouvrage, nous pouvons en égrener 
quelques-unes comme exemples, dans l'ordre chronologique de leur réalisation: 2001, I'Odyssée de l'espace (1968) de Stanley Kubrick, Duel (1971) de Steven Spielberg, La Guerre des étoiles (1977) de George Lucas, Terminator (1984) de James Cameron, Matrix (1999) d'Andy et Lana Wachowski, Alexandre (2004) d'Oliver Stone, Avatar (2009) de James Cameron. Notons que I'auteur distingue deux périodes majeures de la production de ce type de films durant les soixante dernières années. Elles sont scindées par une baisse de régime de ces réalisations cinématographiques à partir du milieu des années 1980 jusqu'au milieu des années 1990. Par conséquent, il postule que "sans être négligeable, les années qui vont de 1988 à 1995 sont d'un intérêt moindre pour [sa] recherche, dans la mesure où la rareté des films de science-fiction et catastrophe peut être tenue pour le signe d'un déficit d'intérêt à l'endroit des tensions générées par le rapport a priori problématique entre l'homme et la technologie " (p. 29). Il précise encore que la sélection des films analysés est induite par plusieurs critères prépondérants, comme:

[...] le succès des films, que ce soit sur le plan de leur accueil public ou critique, mais aussi de la notoriété de la star ou du personnage en tant qu'icône de masculinité, la renommée de l'antagonisme comme contre-type à cet idéal, la présence de figures archétypales propres à la culture nord-américaine, la popularité de leur franchise ou encore le caractère emblématique du film en regard du cycle générique dans lequel il s'inscrit. (p. 29)

De prime abord, la pluralité des critères sélectionnés qui justifient le contenu de la base de données des sources cinématographiques de cette recherche semble répondre à un système quantitatif de référencement plutôt qu'à une sélection qualitative à investiguer pour développer une approche originale de la problématique. Cette large sélection de longs métrages a toutefois pour objectif de permettre à l'auteur de rendre compte, à travers le prisme de l'étude de genre (gender), de l'hétérogénéité des représentations et des discours tenus dans certains films sur le délitement du pouvoir des hommes hétérosexuels issus de la middle class blanche qu'il rattache à l'évolution des technologies. Pour répondre à ce but, Courcoux part de I'hypothèse que la masculinité se construit au gré de contextes socio-culturels qui évoluent dans le temps. Il appuie principalement son propos sur les recherches en gender studies de la sociologue Raewyn Connell qui formalise le concept de masculinité hégémonique. Cette formule désigne, selon elle, "la configuration des pratiques de genre visant à assurer la perpétuation du patriarcat et la domination des hommes sur les femmes ${ }^{5}$. De ce point de vue, la masculinité est un mode relationnel normatif entre les genres qui les régit dans une logique de pouvoir. La masculinité est une "organisation sociale" qui ne constitue pas un objet homogène, mais s'actualise dans des processus, des rapports et des relations qui construisent les genres dans un milieu symbolique. Par conséquent, même si la masculinité est
5 Raewyn Connell, Masculinités. Enjeux sociaux de l'hégémonie, Paris, Amsterdam Editions, 2014, p. 11. 
Id., p. 75.

Ibid.

Id., p. 74.

Id., p. 64.

Judith Butler, Trouble dans le genre (trad. Cynthia Kraus), Paris, La Découverte, 2006 (première publication américaine: 1990), p.39. hégémonique, elle n'est pas faite d'un bloc et sa structure est mouvante en fonction du contexte socio-historique: "L'hégémonie est ainsi une relation historiquement dynamique ${ }^{6}$. Selon Connell, différents régimes de masculinité coexistent et se confrontent en raison du fait que l'ensemble des hommes ne répond pas à la totalité des normes qui font autorité (force physique, pouvoir financier, origine ethnique occidentale, hétérosexualité, etc.). Au sein du cadre général imposé par la masculinité hégémonique, «il existe des rapports spécifiques de domination et de subordination entre des groupes $\mathrm{d}^{\prime}$ hommes ${ }^{7}$. Dès lors, une grande partie de ceux-ci doivent composer stratégiquement entre "subordination", "complicité " et "marginalisation" face au modèle prédominant. Pour la sociologue, la représentation de ce modèle serait en partie relayée par les personnes les plus puissantes, "comme des acteurs de cinéma, voire des figures imaginaires, comme des personnages de films ${ }^{8}$. Elle précise que c'est bien parce que "rares sont les hommes qui collent parfaitement au 'modèles ou qui font preuve de la même force de caractère ou de la même indépendance que John Wayne, Humphrey Bogart ou Clint Eastwood ${ }^{9}$ que la masculinité trouve sa forme par le biais de multiples modalités. Dans le prolongement de cette remarque, pour Judith Butler, la normativité hétérosexuelle ne fonctionne jamais pleinement:

En ce sens, l'hétérosexualité offre des positions sexuelles normatives qui sont intrinsèquement impossibles à incarner, et l'échec répété à s'identifier pleinement et sans incohérence à ces positions révèle que I'hétérosexualité est non seulement une loi obligatoire, mais aussi une comédie inévitable. ${ }^{10}$

Autrement dit, I'homme "performe" sa masculinité à travers un rôle qu'il tient en oscillant de façon plus ou moins contradictoire entre des valeurs sociales imposées qui l'assujettissent et les caractéristiques individuelles qui participent à la construction de son identité en tant que sujet. Dans ce sens, les normes qui régissent le modèle de la masculinité évoluent dans le temps et sa représentation est adaptée en fonction d'un contexte socio-culturel en mutation.

Courcoux convoque donc les recherches de Connell et Butler pour étayer son cadre théorique qui lui permet de structurer son étude des différentes postures de résistance ou d'effritement de la masculinité incarnées par des personnages au cinéma. II résulte de cette analyse que les films de la première période abordée (entre 1968 et 1987) et peut-être plus encore de la seconde (de 1996 à 2014) montrent que l'innovation technologique engendre une phobie chez l'homme en raison des modifications politiques, sociales, économiques et culturelles qu'elle provoque. Ces films révèlent chacun à leur manière la peur générée par l'effondrement de la supériorité de l'homme sur son environnement matériel et social. Cependant, pour l'auteur, à partir de 2015, il y aurait une "réduction des tensions entre masculinité et 
technologie» (p.481) dans les productions du cinéma hollywoodien. Cette réduction de leur rapport conflictuel serait notamment due au fait que la technologie numérique participe pleinement à l'acquisition du pouvoir de certains hommes à la direction d'entreprises les plus puissantes au monde économiquement, comme les GAFA. Ces nouvelles hégémonies portées par la relation entre l'homme et la machine ne s'actualisent pas sur un mode d'opposition, mais de collaboration, soutenu par l'usage de tout un chacun des ordinateurs, des téléphones portables et d'Internet.

À un autre niveau, la réalisation, la production et la diffusion de films à l'aide de la technologie du dispositif cinématographique ont permis d'instaurer un système de pouvoir et de domination entre des hommes (réalisateurs et producteurs) et des femmes (actrices). Dans ce cas, il ne s'agit pas d'un clivage entre "des machines et des hommes", mais d'une connivence qui a parfois engendré des dérives éhontées. Comme nous le savons, ces déviances, dans le milieu du cinéma, ont été dénoncées largement, pour la première fois, en octobre 2017, par le biais du mouvement «Me Too». Ironiquement, ce sont les réseaux sociaux développés avec la technologie informatique qui ont permis à certaines actrices de témoigner à l'échelle mondiale des harcèlements et des agressions qu'elles ont subis. Si, comme le constate Courcoux en guise de conclusion, le rapport entre I'homme et la technologie est aujourd'hui à envisager de manière dialectique au sein du cinéma hollywoodien, depuis la seconde moitié des années 2010, il serait souhaitable que ce dialogue soit opérant tant derrière que devant l'écran dans un souci de reconfiguration et de représentation respectueux de l'égalité entre les genres plutôt que d'être mis au service de nouvelles modalités perverties de domination. 